\section{Enhancing T3 and CAMP responsive gene participation in the thermogenic regulation of fuel oxidation pathways}

\author{
Ampliando a participação dos genes responsivos a T3 e cAMP na \\ regulação da termogênese gerada pelas vias de oxidação biológica
}

Karina Kores Dorsa' ${ }^{1}$ Michelle Venâncio dos Santos' ${ }^{1}$ Magnus R. Dias da Silva'

\begin{abstract}
Objective: We sought to identify glycolysis, glycogenolysis, lipolysis, Krebs cycle, respiratory chain, and oxidative phosphorylation enzymes simultaneously regulated by T3 and cAMP. Materials and methods: We performed in silico analysis of 56 promoters to search for cis-cAMP (CREB) and cis-thyroid (TRE) response elements, considering UCP1, SERCA2 and glyceraldehyde 3-phosphate dehydrogenase as reference. Only regulatory regions with prior in vitro validation were selected. Results: 29/56 enzymes presented potential TREs in their regulatory sequence, and some scored over 0.80 (better predictive value 1): citrate synthase, phosphoglucose isomerase, succinate dehydrogenases A/C, UCP3, UCP2, UCP4, UCP5, phosphoglycerate mutase, glyceraldehyde 3-P dehydrogenase, glucokinase, malate dehydrogenase, acyl-CoA transferase (thiolase), cytochrome a3, and lactate dehydrogenase. Moreover, some enzymes have not yet been described in the literature as genomically regulated byT3. Conclusion: Our results point to other enzymes which may possibly be regulated by T3 and CREB, and speculate their joint roles in contributing to the optimal thermogenic acclimation. Arq Bras Endocrinol Metab. 2010;54(4):381-9
\end{abstract}

\section{Keywords}

Thermogenesis; T3R; CREB; genomic regulation; fuel oxidation

\section{RESUMO}

Objetivo: Identificar enzimas das vias da glicólise, glicogenólise, lipólise, ciclo de Krebs, cadeia respiratória e fosforilação oxidativa possivelmente reguladas porT3 e cAMP. Materiais e métodos: Analisamos 56 genes metabólicos in silico mediante a identificação dos elementos cis de regulação gênica responsivos ao T3 e cAMP (TREs, thyroid response elements e CREs, cAMP response elements), utilizando como referência o promotor da UCP1, SERCA2 e gliceraldeído 3-fosfato desidrogenase. Selecionamos somente os promotores com estudo funcional prévio in vitro. Resultados: 29/56 enzimas apresentaramTREs em suas regiões regulatórias, parte com escore $\geq 0,80$ (melhor valor preditivo 1): citrato sintase, fosfoglucose isomerase, succinato desidrogenase A/C, UCP3, UCP2, UCP4, UCP5, fosfoglicerato mutase, gliceraldeído 3-P desidrogenase, glucoquinase, malato desidrogenase, acil-CoA transferase (tiolase), citocromo a3, e lactato desidrogenase; parte desconhecida como regulada porT3. Conclusão: Os resultados do presente estudo apontam para novos genes regulados porT3 e cAMP e, por conseguinte, sua contribuição na regulação da termogênese. Arq Bras Endocrinol Metab. 2010;54(4):381-9

Descritores

Termogênese; T3R; CREB; regulação gênica; oxidação biológica
1 Universidade Federal de São Paulo (Unifesp), São Paulo, SP, Brazil
Correspondence to: Magnus R. Dias da Silva Laboratório de Endocrinologia Molecular,

Departamento de Bioquímica, Universidade Federal de São Paulo 04044-020 - São Paulo, SP, Brazil magnus.bioq@epm.br

Received on Nov/13/2009 Accepted on Feb/9/2010

\section{INTRODUCTION}

$\mathrm{B}$ ody heat is a by-product of the transformation of chemical energy slowly released during food oxida- tion. This apparent thermodynamic inefficiency keeps our body warm and optimizes the functioning of cells and systems. It is widely known that T3 is one of the key 
mediators of thermogenesis $(1,2)$, especially through the control of transcriptional uncoupling proteins (UCPs) together with the partner CAMP-CREB, cyclic AMP response element binding protein (1). In fact, UCPs optimize the inefficiency of mitochondrial thermogenesis by shifting the gradient of protons from the ATP synthesis to generate heat (3), as the consequence of proton leak amplification. Despite of the recognized role of T3 in generating heat through UCP canonical thermogenic path $(1,4,5)$, little is known about its transcriptional regulation in other metabolic oxidative enzymes. Much less is clear about T3R-CREB transcriptional regulation partnership in orchestrating the fuel oxidation pathways.

Besides the accumulated evidence on T3R-CREB/ UCP thermogenic path (6), we should also consider adding the role of 3,5-diiodo-1-thyronine (T2) and the substrate, or futile cycling contributions to heat generation. In fact, it has been demonstrated that interplay between opposite metabolic routes such as glucose and glucose-6-phosphate and between fructose-6-phosphate and fructose-1,6-diphosphate occurs in man and is directly affected by T3, these substrate cycles contribution may represent a mechanism by which thyroid hormone alters the sensitivity of certain reactions to metabolic signals (7). Moreover, not only T3 but also T2 enhances skeletal muscle mitochondrial thermogenesis by activating pathways involved in the dissipation of the proton-motive force (ATP generation) towards proton leak (heat), the effect being dependent on the presence of free fatty acids inside mitochondria (8). It may also be worth considering further comprehensive overviews on regulation of thermogenesis $(1,9,10)$.

Why search for cis-elements in a set of regulatory genomic sequences? In the last decade a great amount of differentially expressed genes was made available by the widespread use of gene expression profiling, especially from microarray and SAGE analysis, without scrutinizing information about their transcriptional regulation. In fact, these methods only take into account arrays created from cDNA sequences of pooled genes, and most of the transcriptional regulatory signals; however, they are more likely to be in the promoter and enhancer/silencer regions.

Although it has been an arduous task delineating the regulatory region of a gene due to the difficulty in establishing the start and the end of its sequence, promoter sequences corresponding to selected cDNAs can be traced either by exon-mapping or discovered by in silico promoter prediction tools from whole genome sequen- ces available on public databases (11-13), making it more straightforward. The lack of reliable definition of the promoter size has been overcome with new software, but variability in the limits of regulatory regions still remains. Some programs in bioinformatics, for instance, also perform comparative promoter analysis among coexpressed genes, and then identify common transcription regulatory signals (14-16). These smart web-based tools can be applied to help narrow down an enormous amount of expressed genes provided by selecting canonical transcription factors as filters, in addition to sorting them by specific tissues or pathways.

One of the most important transcriptional factors related to thermogenesis is CREB, which is constitutively expressed in the cell. In fact, CREB activity is greatly increased after phosphorylation by cAMP, which works as a cell sensor of a variety of stimuli, including those downstream of adenilate cyclase activation in the cell membrane $(17,18)$. In addition, the TSH receptor is also mainly coupled to the cAMP cascade, in which the cAMP pathway exerts a dual function as it regulates both thyroid hormone production and proliferation of thyroid epithelial cells (19). Interestingly, we have found that several thermometabolic steps can be simultaneously regulated at the transcriptional level by cAMP-CREB and T3/T3R (20-23), in which we postulate whether both factors do really have an intimate transcriptional crosstalk in generating heat.

\section{MATERIALS AND METHODS}

We sought to identify enzymes involved in glycolysis, glycogenolysis, lipid ß-oxidation, Krebs cycle, respiratory chain, and oxidative phosphorylation possibly regulated by $\mathrm{T} 3$ and cAMP. We performed in silico analysis of 56 promoters related to all enzymes involved in the above-mentioned metabolic routes to search for $c i$-thyroid response elements (TRE) together with c-AMP response elements (CRE), initially using the GENOMATIX program (16), available to academic institutions through the website: http://www.genomatix.de/cgi-bi. The latter predicts the best regulatory sequence of a gene based on the degree of conservation between orthologs, classifying it in the gold, silver or bronze groups accordingly with the best evidence for experimentally verified 5' complete transcript, with 5' end confirmed by the software prediction only, and annotated transcript without confirmation for 5' sequence completeness, respectively. 
We have accepted the lowest cutoff threshold obtained from the analysis of the UCPI, SERCA2 and glyceraldehyde 3-phosphate dehydrogenase regulatory regions as reference, since they have been classically demonstrated in the literature as the strongest T3-regulated genes. Indeed, we have only selected regulatory regions with a prior in vitro validation of the promoter, according to evidence made available from the GENOMATIX program termed golden promoter.

Once selected, the promoters were followed with a narrowing second screening using three independent programs PROMO (24), ALIBABA2 (25), and TESS (15), all publicly available through sites: http:// alggen.lsi.upc.es/cgi-bin/promo_v3/promo, http:// www.gene-regulation.com/pub/programs/alibaba2, and http://www.cbil.upenn.edu/cgi-bin/tess. TESS uses human CREB (T00163) or T3R-betal (T00851) as core position factor filters for TRANSFAC strings, accepting 10\% of Maximum Allowable String Mismatch $\left(\mathrm{t}_{\mathrm{mm}}\right)$, a real number $\geq 0$ of $\log$-likelihood ratio score $\left(\mathrm{t}_{\mathrm{s}-\mathrm{a}}\right)$, and the Minimum string length $\left(\mathrm{t}_{\mathrm{w}}\right)$ of 6 . In fact, most of the available programs for predicting transcription factor binding sites use known sites collected from TRANSFAC major matrix, which contains data on transcription factors, their experimentally-proven binding sites, and an array of regulated genes. Its broad compilation of binding sites allows the derivation of positional weight matrices, so it calculates a p-value for each prediction (26).

These programs are able to specifically search for T3R-b cis-element binding sites among all the transcriptional factors on a matrix, grouped within either RXR or CREB family on query regulatory sequences. We have only selected the binding sites for RXRF and CREB that have scored over the defined threshold, assuming a statistically significant $\mathrm{p}$-value $<0.01$ (provided by the program).

\section{RESULTS}

We were able to identify 29 promoters that presented TREs among all 56 transcriptionally-inspected metabolic enzymes as summarized on table 1 . The top 10 enzyme promoters that scored over 0.80 (better predictive value 1) were: citrate synthase, phosphoglucose isomerase, succinate dehydrogenase A/C, UCP3, UCP2, UCP4, UCP5, phosphoglycerate mutase, glycerol 3-phosphate dehydrogenase, glucokinase, malate dehydrogenase, acyl-CoA transferase (thiolase), cytochrome a3, and lactate dehydrogenase. To the best of our knowledge some pulled enzymes have not yet been described in the literature as possibly regulated by T3. Therefore, our results point to more enzymes genomically regulated by $\mathrm{T} 3$, possibly broadening its role in the metabolic process of thermogenesis. See table 1 for more details regarding enzyme metabolic pathway and cell localization, their NCBI gene identification, the size of the promoter used for studying, the Initial screening by GENOMATIX program when the score was set on $\mathrm{p}<0.01$, the second screening using a more specific filter for CRE and TRE by PROMO programs of bioinformatics.

\section{DISCUSSION}

Our study extends the participation of $\mathrm{T} 3$ regulation to many metabolic enzymes, adding to the understanding of fuel oxidation enzymatic steps for generating heat, as summarized on figure 1 . We also demonstrate the usefulness of several in silico available resources in searching for potential promoters and for better fishing of transcriptional signals. By using these bioinformatics resources we were able to not only filter for specific transcriptional family factors, but also to compare promoters for common ones. Part of the usefulness of the work described here lies in the ability of rapid screening of gene expression driven by hormonal control. As a result, we observed marked overlapping signals between T3R and CREB (trans-elements) signals, in searching for their cis-elements (TRE and CRE). From a theoretical viewpoint, we speculate that these transcriptional factors play adjunct roles in orchestrating some oxidative enzymes in the same thermometabolic routes.

Most enzymes regulated by T3R/CREB identified in our study are well known as key steps of fuel metabolism. However, some of them have not yet been described in the literature as being regulated by T3, of which the following stand out: Phosphoglucose Isomerase (Figure 1.2/1.3), Succinate Dehydrogenase C and Succinate Dehydrogenase A (Figure 1.6), NDUFV3/V1/V2 Flavoprotein NADH Dehydrogenase 1 and COX4Il - Cytochrome a / a3 (Figure 1.7/1.8), Uncoupling Protein 5 (Figure 1.7/1.9), Phosphoglucomutase (Figure 1.3), ACATl acetyl-Coenzyme A acetyltransferase 1 (Thiolase 1 ) and ACAT2 acetylCoenzyme A acetyltransferase 2 (Thiolase 2) (Figure 1.1/1.5). These verified enzymes are particularly discussed as follows. 
Table 1. In silico screening of TRE (RXRF/T3R-b) and CRE (CREB/CAMP) cis-regulatory elements of metabolic enzyme

\begin{tabular}{|c|c|c|c|c|c|c|c|c|c|c|c|}
\hline \multirow[b]{2}{*}{$\mathbf{N}^{\mathbf{0}}$} & \multirow[b]{2}{*}{$\begin{array}{l}\text { Metabolic pathway } \\
\text { enzyme }\end{array}$} & \multirow[b]{2}{*}{$\begin{array}{c}\text { Cell } \\
\text { localization }\end{array}$} & \multirow[b]{2}{*}{$\begin{array}{c}\text { NCBI } \\
\text { gene ID }\end{array}$} & \multicolumn{5}{|c|}{ Initial screening by GENOMATIX software } & \multicolumn{3}{|c|}{$\begin{array}{l}\text { Second screening - filtering for } \\
\text { T3R-b cis specific sites }\end{array}$} \\
\hline & & & & $\begin{array}{l}\text { Promoter } \\
\text { size (bp) }\end{array}$ & $\begin{array}{l}\text { Total No } \\
\text { of CREB } \\
\text { cis sites }\end{array}$ & $\begin{array}{c}\text { CREB (cAMP) score, } \\
\mathrm{p}<0.01\end{array}$ & $\begin{array}{l}\text { Total } \mathrm{N}^{\circ} \\
\text { of RXRF } \\
\text { cis sites }\end{array}$ & $\begin{array}{c}\text { RXRF (T3R-b) score, } \\
\mathrm{p}<0.01\end{array}$ & \begin{tabular}{|l|} 
Total No \\
PROMO \\
software
\end{tabular} & \begin{tabular}{|c|} 
Total No \\
ALIBABA2 \\
software
\end{tabular} & \begin{tabular}{|l} 
Total No \\
TESS \\
software
\end{tabular} \\
\hline & Glycolysis & & & & & & & & & & \\
\hline 1 & Hexokinase & Cytosolic & 3098 & 602 & 2 & $2 s l-0.919 / 0.915$ & 3 & $2 s l-0.853 / 0.816$ & 3 & 1 & 0 \\
\hline 2 & Glucokinase & Cytosolic & 2646 & 668 & 0 & - & 1 & 1sl -0.859 & 2 & 1 & 0 \\
\hline 3 & $\begin{array}{l}\text { Phosphoglucose } \\
\text { Isomerase }\end{array}$ & Cytosolic & 2821 & 601 & 4 & $3 s \mathrm{~s}-0.771 / 0.912 / 0.944$ & 2 & $2 \mathrm{sl}-0.906 / 0.8$ & 3 & 2 & 0 \\
\hline 4 & Phosphofructokinase 1 & Cytosolic & 5211 & 669 & 1 & $1 s \mid-0.888$ & 1 & - & 0 & 1 & 1 \\
\hline 5 & Aldolase A & Cytosolic & 226 & 668 & 0 & - & 0 & - & 3 & 0 & 0 \\
\hline 6 & $\begin{array}{l}\text { Triosephosphate } \\
\text { Isomerase }\end{array}$ & Cytosolic & 7167 & 911 & 0 & - & 3 & $\begin{array}{l}3 \mathrm{~s} /-0.771 / 0.806 / \\
0.904\end{array}$ & 0 & 1 & 0 \\
\hline 7 & \begin{tabular}{|c|} 
Gluceraldehyde 3 \\
Phosphate Dehydrogenase
\end{tabular} & Cytosolic & 26330 & 718 & 13 & $\begin{array}{c}\text { 10sl - } 0.968 / 0.98 / 1.0 / \\
1.0 / 0.974 / 0.931 / 0.935 \\
/ 0.918 / 0.722 / 0.891\end{array}$ & 4 & $\begin{array}{l}3 \mathrm{sl}-0.864 / 0.816 / \\
0.76\end{array}$ & 4 & 0 & 1 \\
\hline 8 & Phosphoglyrate Kinase & Cytosolic & 5232 & 601 & 2 & $1 \mathrm{sl}-0.86$ & 5 & 3st - 0.76/0.769/0.791 & 3 & 1 & 0 \\
\hline 9 & Phosphoglyrate Mutase & Cytosolic & 5224 & 799 & 1 & - & 3 & $\begin{array}{c}3 \mathrm{~s} /-0.795 / 0.807 / \\
0.869\end{array}$ & 2 & 0 & 0 \\
\hline 10 & Enolase & Cytosolic & 2027 & 612 & 4 & $\begin{array}{c}4 \mathrm{sl}-0.934 / 0.968 / 0.926 \\
/ 0.917\end{array}$ & 4 & $\begin{array}{c}4 \mathrm{~s} /-0.796 / 0.793 / \\
0.77 / 0.786\end{array}$ & 4 & 2 & 0 \\
\hline 11 & Pyruvate Kinase & Cytosolic & 5313 & 648 & 3 & $2 s \mathrm{sl}-0.846 / 0.875$ & 2 & - & 2 & 0 & 0 \\
\hline \multirow[t]{2}{*}{12} & Lactate Dehydrogenase & Cytosolic & 3939 & 824 & 9 & $\begin{array}{c}9 \mathrm{gl}-0.909 / 0.97 / 1.0 / \\
1.0 / 0.976 / 0.888 / 0.894 \\
/ .0911 / 0.817\end{array}$ & 2 & $2 s \mathrm{~s}-0.842 / 0.887$ & 1 & 0 & 1 \\
\hline & Krebs Cycle & & & & & & & & & & \\
\hline 13 & $\begin{array}{c}\text { Pyruvate Dehydrogenase } \\
\text { (lipoamide) Alpha } 1\end{array}$ & MM & 5160 & 777 & 4 & $3 \mathrm{sl}-0.848$ / 0.932 / 1.0 & 1 & $1 \mathrm{~s} \mid-0.847$ & 1 & 1 & 2 \\
\hline 14 & Aconitase & MM & 48 & 663 & 0 & - & 3 & $2 \mathrm{sl}-0.81 / 0.872$ & 0 & 1 & 0 \\
\hline 15 & Isocitrate Dehydrogenase & MM & 3420 & 673 & 2 & - & 1 & - & 1 & 2 & 0 \\
\hline 16 & $\begin{array}{c}\text { Oxoglutarate } \\
\text { (alpha-ketoglutarate) } \\
\text { Dehydrogenase }\end{array}$ & MM & 4967 & 601 & 11 & $\begin{array}{c}11 s l-0.854 / 1.0 / 1.0 / \\
0.945 / 0.962 / 0.88 / 0.9 / \\
0.926 / 0.921 / 0.892 / \\
0.908\end{array}$ & 2 & $2 \mathrm{sl}-0.774 / 0.705$ & 0 & 1 & 10 \\
\hline 17 & $\begin{array}{c}\text { Succinyl-CoA Synthetase } \\
\text { Subunit Alpha }\end{array}$ & MM & 8802 & 602 & 0 & - & 2 & $1 \mathrm{sl}-0.956$ & 1 & 0 & 0 \\
\hline 18 & $\begin{array}{c}\text { Succinate Dehydrogenase } \\
\text { C }\end{array}$ & MM & 6391 & 614 & 5 & $\begin{array}{c}4 \mathrm{sl}-0.906 / 0.922 / 0.926 \\
/ 0.9\end{array}$ & 0 & - & 1 & 2 & 1 \\
\hline 19 & $\begin{array}{l}\text { SDHA Succinate } \\
\text { Dehydrogenase A }\end{array}$ & MM & 6389 & 611 & 1 & $1 \mathrm{sl}-0.844$ & 2 & $2 s \mathrm{~s}-0.771 / 0.802$ & 1 & 1 & 0 \\
\hline 20 & \begin{tabular}{|c|} 
Succinate Dehydrogenase \\
D
\end{tabular} & MM & 6392 & 650 & 0 & - & 4 & $\begin{array}{c}4 \mathrm{~s} \mid-0.831 / 0.877 / \\
0.808 / 0.752\end{array}$ & 2 & 0 & 0 \\
\hline 21 & Fumarate Hydratase & MM & 2271 & 601 & 2 & $2 s \mathrm{~s}-0.879 / 0.908$ & 0 & - & 2 & 0 & 1 \\
\hline \multirow[t]{2}{*}{22} & Malate Dehydrogenase & $\mathrm{MM}$ & 4190 & 1141 & 0 & - & 5 & \begin{tabular}{|c|}
$5 \mathrm{sl}-0.854 / 0.75 /$ \\
$0.799 / 0.803 / 0.809$ \\
\end{tabular} & 3 & 1 & 2 \\
\hline & Respiratory Chain & & & & & & & & & & \\
\hline 23 & $\begin{array}{l}\text { NDUFV3 flavoprotein } \\
\text { NADH dehydrogenase }\end{array}$ & $\begin{array}{l}\text { Complex I } \\
\text { (MMI) }\end{array}$ & 4731 & 670 & 2 & $2 s \mathrm{~s}-0.976 / 0.998$ & 2 & $1 \mathrm{sl}-0.799$ & 3 & 0 & 2 \\
\hline 24 & $\begin{array}{l}\text { NDUFV1 flavoprotein } \\
\text { NADH dehydrogenase }\end{array}$ & $\begin{array}{l}\text { Complex I } \\
\text { (MMI) }\end{array}$ & 4723 & 754 & 4 & $2 s \mathrm{~s}-0.941 / 0.983$ & 3 & $2 \mathrm{sl}-0.894 / 0.814$ & 3 & 2 & 5 \\
\hline \multirow[t]{2}{*}{25} & $\begin{array}{l}\text { NDUFV2 flavoprotein } \\
\text { NADH dehydrogenase }\end{array}$ & $\begin{array}{l}\text { Complex I } \\
\text { (MMI) }\end{array}$ & 4729 & 668 & 4 & $2 s \mathrm{~s}-0.918 / 0.926$ & 2 & $2 \mathrm{sl}-0.802 / 0.804$ & 3 & 0 & 1 \\
\hline & \begin{tabular}{|c|} 
SDHA flavoprotein \\
succinate dehydrogenase
\end{tabular} & $\begin{array}{l}\text { Complex II } \\
\text { (MMI) }\end{array}$ & 6389 & 611 & 1 & $1 \mathrm{sl}-0.844$ & 2 & $2 s \mathrm{~s}-0.771 / 0.802$ & 1 & 1 & 0 \\
\hline 26 & $\begin{array}{c}\text { GPD1 (flavoprotein) } \\
\text { glycerol 3-phosphate } \\
\text { dehydrogenase (soluble) }\end{array}$ & External MMI & 2819 & 611 & 1 & $1 \mathrm{sl}-0.758$ & 2 & $1 s \mathrm{l}-0.761$ & 1 & 0 & 2 \\
\hline 27 & $\begin{array}{l}\text { GPD2 (flavoprotein) } \\
\text { glycerol 3-phosphate } \\
\text { dehydrogenase } \\
\text { (mitochondrial) }\end{array}$ & External MMI & 2820 & 912 & 4 & $3 \mathrm{sl}-0.888$ / 0.834 / 0.906 & 3 & $\begin{array}{c}3 \mathrm{sl}-0.725 / 0.762 / \\
0.936\end{array}$ & 2 & 3 & 2 \\
\hline 28 & $\begin{array}{c}\text { CYBA cytochrome b-245, } \\
\text { alpha polypeptide }\end{array}$ & $\begin{array}{l}\text { Complex III } \\
\text { (MMI) }\end{array}$ & 1535 & 662 & 2 & $2 \mathrm{sl}-0.992 / 0.908$ & 2 & $1 \mathrm{sl}-0.779$ & 2 & 0 & 2 \\
\hline 29 & $\begin{array}{l}\text { UCRC ubiquinol- } \\
\text { cytochrome c reductase } \\
\text { complex }\end{array}$ & $\begin{array}{l}\text { Complex III } \\
\text { (MMI) }\end{array}$ & 29796 & 630 & 1 & 1sl -0.888 & 1 & $1 \mathrm{sl}-0.903$ & 2 & 0 & 0 \\
\hline
\end{tabular}


Table 1. Continuation

\begin{tabular}{|c|c|c|c|c|c|c|c|c|c|c|c|}
\hline \multirow[b]{2}{*}{$\mathbf{N}^{\circ}$} & \multirow[b]{2}{*}{$\begin{array}{l}\text { Metabolic pathway } \\
\text { enzyme }\end{array}$} & \multirow[b]{2}{*}{$\begin{array}{c}\text { Cell } \\
\text { localization }\end{array}$} & \multirow[b]{2}{*}{$\begin{array}{c}\text { NCBI } \\
\text { gene ID }\end{array}$} & \multicolumn{5}{|c|}{ Initial screening by GENOMATIX software } & \multicolumn{3}{|c|}{$\begin{array}{l}\text { Second screening - filtering for } \\
\text { T3R-b cis specific sites }\end{array}$} \\
\hline & & & & $\begin{array}{l}\text { Promoter } \\
\text { size (bp) }\end{array}$ & $\begin{array}{l}\text { Total No } \\
\text { of CREB } \\
\text { cis sites }\end{array}$ & $\begin{array}{c}\text { CREB (cAMP) score, } \\
\mathrm{p}<0.01\end{array}$ & $\begin{array}{l}\text { Total No } \\
\text { of RXRF } \\
\text { cis sites }\end{array}$ & $\begin{array}{c}\text { RXRF (T3R-b) score, } \\
\mathrm{p}<0.01\end{array}$ & $\begin{array}{l}\text { Total No } \\
\text { PROMO } \\
\text { software }\end{array}$ & $\begin{array}{l}\text { Total No } \\
\text { ALIBABA2 } \\
\text { software }\end{array}$ & $\begin{array}{l}\text { Total No }^{\circ} \\
\text { TESS } \\
\text { software }\end{array}$ \\
\hline 30 & $\begin{array}{l}\text { COX4l1 - cytochrome a / } \\
\text { a3 subunit IV isoform } 1\end{array}$ & $\begin{array}{c}\text { Complex IV } \\
\text { (MMI) }\end{array}$ & 1327 & 786 & 1 & $1 \mathrm{sl}-0.921$ & 1 & $1 \mathrm{sl}-0.793$ & 4 & 1 & 0 \\
\hline 31 & $\begin{array}{l}\text { SLC25A31 solute carrier } \\
\text { family } 25 \text {, adenine } \\
\text { nucleotide translocator } \\
\text { member } 31\end{array}$ & $\mathrm{MMI}$ & 83447 & 1049 & 9 & $\begin{array}{c}\text { 6sl - } 0.959 \text { / } 0.935 / 0.911 \\
/ 0.917 / 0.914 / 0.895\end{array}$ & 5 & $\begin{array}{c}3 \mathrm{sl}-0.765 / 0.762 / \\
0.762\end{array}$ & 3 & 0 & 9 \\
\hline 32 & $\begin{array}{l}\text { SLC25A6 solute carrier } \\
\text { family 25, adenine } \\
\text { nucleotide translocator } \\
\text { member } 6\end{array}$ & $\mathrm{MMI}$ & 293 & 772 & 4 & $3 s \mathrm{sl}-0.825 / 0.88 / 0.727$ & 3 & $2 s \mathrm{~s}-0.898 / 0.828$ & 0 & 3 & 2 \\
\hline 33 & $\begin{array}{c}\text { SLC 25A4 solute carrier } \\
\text { family 25, adenine } \\
\text { nucleotide translocator } \\
\text { member } 4\end{array}$ & MMI & 291 & 740 & 0 & - & 2 & $1 \mathrm{sl}-0.881$ & 4 & 1 & 0 \\
\hline 34 & $\begin{array}{c}\text { GPD1 glycerol } \\
\text { 3-phosphate } \\
\text { dehydrogenase } 1 \text { (soluble) }\end{array}$ & Cytosolic & 2819 & 611 & 1 & $1 \mathrm{sl}-0.758$ & 2 & $1 \mathrm{~s} \mid-0.761$ & 1 & 0 & 2 \\
\hline 35 & $\begin{array}{c}\text { MDH1 malate } \\
\text { dehydrogenase } 1\end{array}$ & MMI (LMA) & 4190 & 1141 & 0 & - & 5 & $\begin{array}{c}5 \mathrm{sl}-0.854 / 0.75 / \\
0.799 / 0.803 / 0.809\end{array}$ & 6 & 1 & 2 \\
\hline 36 & $\begin{array}{c}\text { GOT2 mitochondrial } \\
\text { aspartate aminotransferase } 2\end{array}$ & MMI (LMA) & 2806 & 734 & 7 & \begin{tabular}{|c|}
$5 \mathrm{~s}-0.968 / 0.949 / 0.993$ \\
$/ 0.911 / 0.896$
\end{tabular} & 4 & $2 s \mathrm{~s}-0.804 / 0.769$ & 1 & 2 & 4 \\
\hline 37 & $\begin{array}{l}\text { G0T1 aspartate } \\
\text { transferase } 2\end{array}$ & Cytosolic & 2805 & 736 & 2 & $1 \mathrm{sl}-0.885$ & 1 & $1 \mathrm{~s} \mid-0.818$ & 3 & 1 & 0 \\
\hline 38 & CS citrate synthase & $\mathrm{MMI}$ & 1431 & 753 & 5 & $2 \mathrm{sl}-0.886 / 0.823$ & 6 & $\begin{array}{l}5 \mathrm{sl}-0.872 / 0.823 / \\
0.847 / 0.971 / 0.888\end{array}$ & 2 & 2 & 6 \\
\hline 39 & ACLY citrate lyase & Cytosolic & 47 & 749 & 0 & - & 0 & - & 1 & 0 & 2 \\
\hline 40 & $\begin{array}{l}\text { UCP1 protein uncoupling } \\
1\end{array}$ & MMI (MBF) & 7350 & 726 & 2 & $2 s \mathrm{~s}-0.911 / 0.881$ & 5 & $\begin{array}{l}4 \mathrm{sl}-0.804 / 0.804 / \\
0.76 / 0.905\end{array}$ & 4 & 2 & 3 \\
\hline 41 & $\begin{array}{l}\text { UCP2 protein uncoupling } \\
2\end{array}$ & $\begin{array}{l}\text { rather than } \\
\text { MBF }\end{array}$ & 7351 & 1065 & 4 & $1 s \mid-0.901$ & 4 & $\begin{array}{c}3 \mathrm{sl}-0.871 / 0.81 / \\
0.886\end{array}$ & 1 & 0 & 8 \\
\hline 42 & $\begin{array}{l}\text { UCP3 protein uncoupling } \\
3\end{array}$ & $\begin{array}{l}\text { rather than } \\
\text { MBF }\end{array}$ & 7352 & 1137 & 3 & $\begin{array}{c}3 \mathrm{sl}-0.831 / 0.823 / \\
0.723\end{array}$ & 6 & $\begin{array}{l}5 \mathrm{sl}-0.874 / 0.804 / \\
0.88 / 0.874 / 0.791\end{array}$ & 7 & 3 & 1 \\
\hline 43 & $\begin{array}{l}\text { UCP4 protein uncoupling } \\
4\end{array}$ & $\begin{array}{l}\text { rather than } \\
\text { MBF }\end{array}$ & 9481 & 602 & 1 & $1 \mathrm{sl}-0.876$ & 4 & $\begin{array}{l}3 \mathrm{sl}-0.862 / 0.845 / \\
0.8\end{array}$ & 2 & 0 & 1 \\
\hline \multirow[t]{2}{*}{44} & \begin{tabular}{|l} 
UCP5 protein uncoupling \\
5 \\
\end{tabular} & $\begin{array}{l}\text { rather than } \\
\text { MBF }\end{array}$ & 9016 & 1183 & 3 & $3 \mathrm{sl}-0.934 / 0.91 / 0.918$ & 4 & $\begin{array}{c}3 \mathrm{sl}-0.778 / 0.809 / \\
0.82 \\
\end{array}$ & 6 & 3 & 2 \\
\hline & Glycogenolysis & & & & & & & & & & \\
\hline 45 & Glycogen phosphorylase & Cytosolic & 5834 & 964 & 6 & $\begin{array}{l}3 \mathrm{sl}-0.925 / 0.918 / \\
0.915\end{array}$ & 2 & $2 s \mathrm{~s}-0.809 / 0.818$ & 2 & 0 & 0 \\
\hline 46 & Phosphoglucomutase & Cytosolic & 5239 & 1074 & 3 & $2 \mathrm{sl}-0.961 / 0.93$ & 2 & $1 \mathrm{sl}-0.798$ & 3 & 1 & 0 \\
\hline 47 & $\begin{array}{c}\text { Glucose - } 6 \text { - Phosphate } \\
\text { Translocase }\end{array}$ & Cytosolic & 2542 & 601 & 1 & $1 \mathrm{sl}-0.743$ & 2 & $1 \mathrm{sl}-0.83$ & 2 & 0 & 0 \\
\hline \multirow[t]{2}{*}{48} & Glucose - 6 - Phosphatase & Cytosolic & 57818 & 744 & 2 & 1sl - 0.878 & 1 & - & 1 & 1 & 0 \\
\hline & $\beta$-0xidation & & & & & & & & & & \\
\hline 49 & $\begin{array}{l}\text { Acyl CoA Synthetase } \\
\text { (ACSL 6) }\end{array}$ & Cytosolic & 23305 & 775 & 2 & $2 s \mathrm{~s}-0.937 / 0.914$ & 4 & $\begin{array}{c}3 \mathrm{sl}-0.862 / 0.91 / \\
0.729\end{array}$ & 1 & 1 & 0 \\
\hline 50 & $\begin{array}{l}\text { Acyl CoA Synthetase } \\
\text { (ACSL 1) }\end{array}$ & Cytosolic & 2180 & 601 & 1 & - & 1 & $1 \mathrm{~s} \mid-0.799$ & 0 & 1 & 0 \\
\hline 51 & Carnitine Acyl Transferase & $\begin{array}{l}\text { Cytosolic / } \\
\text { MM }\end{array}$ & 1384 & 601 & 1 & $1 \mathrm{sl}-0.723$ & 2 & $1 \mathrm{sl}-0.816$ & 1 & 2 & 3 \\
\hline 52 & Acyl CoA Dehydrogenase & MM & 28976 & 803 & 13 & \begin{tabular}{|c|}
$11 s l-0.86 / 0.994 / 1.0 /$ \\
$1.0 / 1.0 / 0.985 / 0.935 /$ \\
$0.912 / 0.935 / 0.892 / 0.89$
\end{tabular} & 0 & - & 1 & 0 & 0 \\
\hline 53 & Enoyl CoA Hydratase & MM & 1891 & 832 & 7 & $\begin{array}{l}5 s l-0.844 / 0.902 / \\
0.922 / 0.893 / 0.744\end{array}$ & 1 & $1 \mathrm{sl}-0.832$ & 1 & 2 & 0 \\
\hline 54 & $\begin{array}{l}\beta \text { Hydroxyacyl CoA } \\
\text { Dehydrogenase }\end{array}$ & MM & 3030 & 601 & 3 & $2 \mathrm{sl}-0.98 / 0.968$ & 3 & $1 \mathrm{~s} \mid-0.773$ & 3 & 0 & 0 \\
\hline 55 & Thiolase 1 & MM & 38 & 679 & 6 & $\begin{array}{c}5 \mathrm{sl}-0.988 / 0.927 / 0.837 \\
/ 0.892 / 0.924\end{array}$ & 3 & $\begin{array}{l}3 \mathrm{sl}-0.808 / 0.775 / \\
0.706\end{array}$ & 2 & 2 & 0 \\
\hline 56 & Thiolase 2 & MM & 39 & 733 & 3 & $3 s \mathrm{~s}-0.985 / 0.9 / 0.843$ & 1 & - & 2 & 1 & 0 \\
\hline
\end{tabular}

NCBI Gene identification number (ID); Mitochondrial brown fat (MBF); Cis-element sites (sl); mitochondrial membrane (MM); number of recognized sites ( $\left(\mathrm{N}^{\circ}\right)$. 


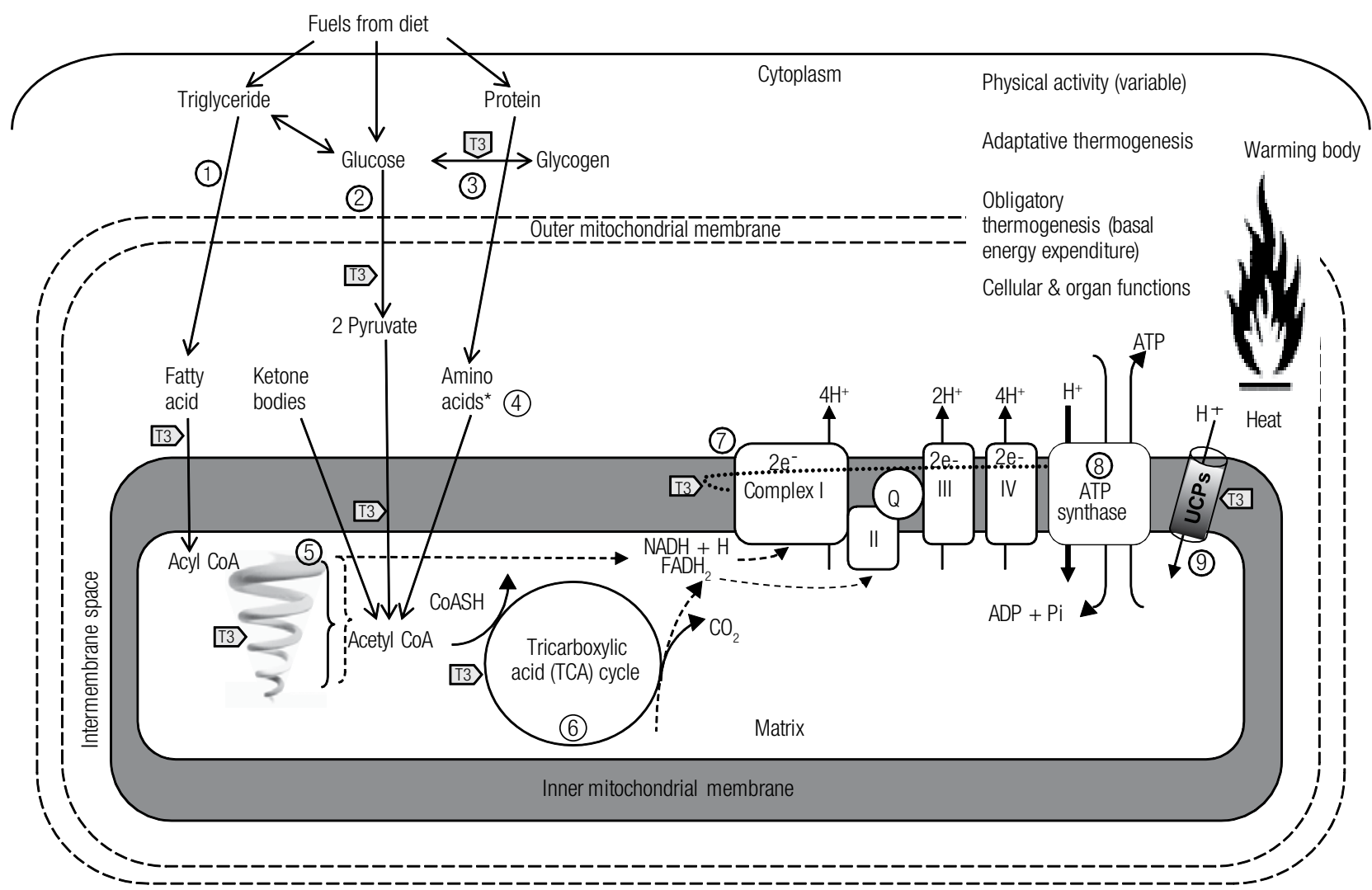

Figure 1. Schematic representation of the interplay between fuel oxidation routes and thermogenesis. 1,5-lipid $\beta$-oxidation, 2-glycolysis, 3-glycogenolysis, 4-proteolysis, 6-Krebs cycle, 7-respiratory chain, 8-oxidative phosphorylation and $\mathbf{9}$-uncoupling protein-meditated proton leak are potentially regulated by $\mathrm{T} 3$ on promoting heat generation. * Glycogenic amino acids.

Glucose phosphate isomerase (GPI, Gene ID: 2821 ) is located on $19 \mathrm{q} 13.1$ and belongs to the GPI family, which members encode multifunctional phosphoglucose isomerase proteins involved in energy pathways. We can expect that T3R/CREB regulation of GPI might increase glucose oxidation, thus generating more heat. In fact, GPI works as a dimeric enzyme that catalyzes the reversible isomerization of glucose6-phosphate and fructose-6-phosphate, so in the cytoplasm, the gene product is involved in glycolysis and gluconeogenesis (27). Interestingly, GPI seems to work outside the cell as a neurotrophic factor for spinal and sensory neurons. Mild defects in this gene cause nonspherocytic hemolytic anemia, while its severe enzymatic deficiency can be associated with hydrops fetalis and immediate neonatal death associated with neurological impairment $(28,29)$. GPI is differentially induced by a hypoxic environment as a transient adaptative response (30), as well as related to regulating cell proliferation, whereas its overexpression may increase in vitro neoplastic transformation (31).
Succinate dehydrogenase complex subunit C (SDHC, Gene ID: 6391) and succinate dehydrogenase complex subunit A (SDHA, Gene ID: 6389) have their genes located on 1q23.3 and 5pl5, respectively. Each gene encodes one of four nuclear-encoded subunits that comprise succinate dehydrogenase, also known as the mitochondrial complex II, where other subunits of the complex anchor, thus forming the major catalytic core of succinate-ubiquinone oxidoreductase, placed in the inner mitochondrial membrane. In addition to participating in the aerobic respiratory chain of mitochondria, it is also a key enzyme complex of the tricarboxylic acid cycle (32). SDHC mutations have been associated with paragangliomas (33). Since alternatively spliced transcript variants of these genes have been described, we may also speculate if T3R/CREB would contribute to one of these transcriptional fates, especially if variations in basal metabolic rate and in climate are taken into account.

NADH dehydrogenase (ubiquinone) flavoprotein 1 , also termed as NDUFVI gene (Gene ID: 4723) encodes the $51-\mathrm{kD}$ subunit of complex I $(\mathrm{NADH}$ : ubiquinone 
oxidoreductase) of the mitochondrial respiratory chain and it is located on 1lq13. Microarray analysis reveals that NDUFVI is one of the six downregulated genes (among 297 genes) when obese and diabetic patients are fed with high fat diet (34). Interestingly each one of the six genes is known to be crucial for oxidative phosphorylation in the skeletal muscle; all of them work on regulation steps quite close to the terminal generation of ATP store. Thus, it is not surprising that T3R/CREB could transcriptionally modulate this metabolic route.

Cytochrome c oxidase (COX4II, Gene ID: 1327) encodes the nuclear-encoded subunit IV isoform 1 the terminal enzyme of the mitochondrial respiratory chain. It is part of a multi-subunit enzyme complex that couples the transfer of electrons from cytochrome $\mathrm{c}$ to molecular oxygen and contributes to a proton electrochemical gradient across the inner mitochondrial membrane. The whole complex consists of 13 mitochondrialand nuclear-encoded subunits, being COX4II located on 16q22-qter. In fact, this gene is genomically placed at the 3 ' of the NOC4 (neighbor of COX4II) gene in a head-to-head orientation, and curiously shares the promoter with it. Thus, it could be hypothesized that both genes are under T3R/CREB genome regulation as well as other mitochondrial enzymes pointed out in our in silico screening. Therefore, we suggest that this group of oxidation enzymes is potentially influenced by thyroid status, orchestrating the tone of metabolism either by the increase of thermodynamic chemical energy or by the increase of proton leak - a thermogenin (UCP) regulating role. Indeed, manipulating COX4II expression on cytochrome c oxidase activity, terminal ATP production, oxygen consumption, and reactive oxygen species generation indicates that this subunit switch is a homeostatic response which optimizes the efficiency of respiration at different oxygen concentrations, especially during temporary muscle hypoxia (35).

Another T3R/CREB possibly regulated enzyme is the mitochondrial uncoupling protein 5 (UCP5), which is a member of the larger family of mitochondrial anion carrier proteins (MACP) (36). The UCP5 gene, also termed SLC25Al4 - a brain mitochondrial solute carrier family 25 , member 14 (Gene ID: 9016) is located in Xq24. As other UCPs, it deviates oxidative phosphorylation from ATP synthesis with energy released as heat, also referred to as the mitochondrial proton leak. Moreover, UCPs facilitate the transfer of anions from the inner to the outer mitochondrial membrane and the return transfer of protons from the outer to the inner mitochondrial membrane, therefore altering $\mathrm{pH}$ gradient. The regulatory role of $\mathrm{T} 3$ on temperature acclimation has been postulated based on the observation of increased UCP3 expression in the skeletal muscle of rats kept over 8 weeks up to $8^{\circ} \mathrm{C}$, and that UCP3 expression is decreased in rats acclimated to $30^{\circ} \mathrm{C}$. Similar findings were verified in hyperthyroid rats compared with euthyroid controls (37).

Phosphoglucomutase 5 (PGM5, Gene ID: 5239) is a phosphotransferase enzyme involved in the interconversion of glucose-1-phosphate and glucose-6phosphate, which has its gene located on $9 \mathrm{ql} 3$ (38). Its activity is essential in the formation of carbohydrates from glucose-6-phosphate and in the formation of glucose-6-phosphate from galactose and glycogen. In fact, it plays a key role in the control of the metabolism towards glycolysis and gluconeogenesis, directing the biochemical fate according to the availability of energy stored as $\operatorname{ATP}(39,40)$.

In addition, the enzymes involved in lipid metabolism -acetyl-Coenzyme A acetyltransferase 1 (or acetoacetyl Coenzyme A thiolase 1, ACAT1, Gene ID: 38) and acetyl-Coenzyme A acetyltransferase 2 (or acetoacetyl Coenzyme A thiolase 2, ACAT2 Gene ID: 39) were also pulled through the screening. They have their genes located on 11q22.3-q23.1 and 6q25.3, respectively. The first encodes a mitochondrial enzyme that catalyzes the reversible formation of acetoacetyl-CoA from two molecules of acetyl-CoA, whereas the second gene encodes the cytosolic enzyme (thiolase 2). It has been suggested that ACAT reflects the metabolic status of the cell by the availability of acetyl molecules likely involved in insulin-mediated regulation (41). As described for cytochrome P450 expression (42), we could suggest that these genes are similarly involved in the T3 regulation of metabolism, even though further in vitro evidence is needed to support our initial insight on the T3R/CREB regulatory-participation hypothesis.

In accordance to our in silico findings, other in vitro evidence has indicated that T3-binding alters the conformation of the thyroid hormone receptor in such a way as to release the corepressor complex (inhibitory) and engage a coactivator complex that comprises multiple histone acetyltransferases, including a steroid receptor family coactivator, a p300/CREB-binding proteinassociated factor (PCAF), and a CREB binding protein (CBP) (2). Taken all together, we were able to assemble this mechanism of partnership as illustrated in figure 2. We also warn that cis-elements for T3R and CREB on regulatory genomic sequences can still be located 


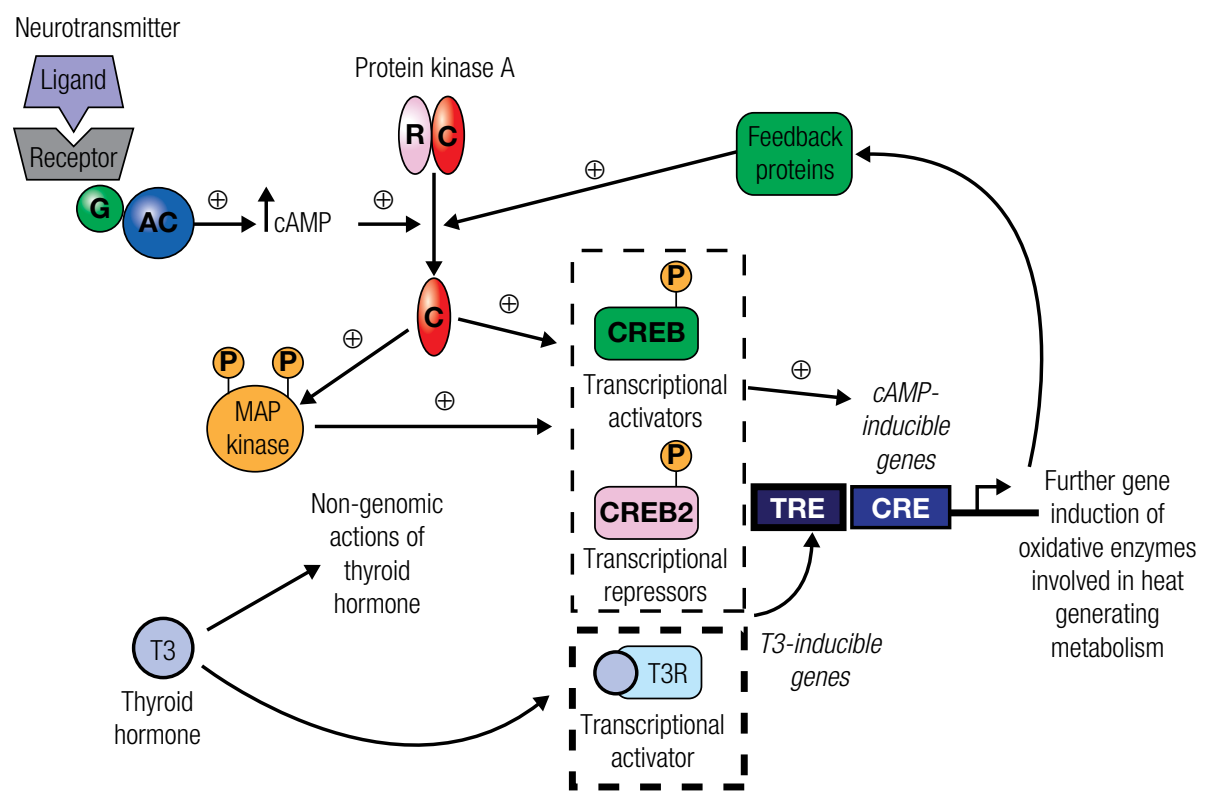

Figure 2. Assembly mechanism of T3R-CREB gene expression regulation of oxidative enzymes involved in heat generating metabolism.

a further $1.2 \mathrm{~Kb}$ upstream of the potential promoter, even though the GENOMATIX program used in our study predicts the best sequence based on the conservation between orthologs, termed as golden promoter. Furthermore, all nucleotide sequences analyzed can be provided when requested to the authors.

In summary, our study identifies more enzymes in the complex spectrum of T3 genomic regulation over the existing ones. The abundance of functional T3-regulated promoters throughout all the metabolic pathways, either alone or with cAMP co-regulation, suggests that the contribution of the thyroid hormone to phenotypic thermogenesis variation and acclimation is likely to be appreciable. This in silico prediction is underscored by the additional impact of either the overt or subclinical, hyper- or hypothyroidism condition (43-45) on gene expression variation, related to thermogenesis. Therefore, this prediction sheds light on how thyroid hormone may influence the basal metabolic tune. It is particularly owing to such screening, and perhaps to certain associated roles (T3R/CREB) found in this work, that efforts are constantly being made, despite the well grounded hypothesis of the major T3-control of UCP expression, to validate in vitro, as yet unknown or unconfirmed joint roles in regulation, as the oxidative enzymes involved in heat generating metabolism.

Acknowledgements: we would like to thank Rui M. B. Maciel (Unifesp) and José Olavo de F. Júnior (Unifesp) and Denise
Carvalho (UFRJ) for helpful discussions and scientific inspiration, and also the technicians of the Department of Biochemistry (Sônia Montanaro) and the Laboratory of Molecular Endocrinology (Teresa Kasamatsu, Ilda Kunni and Giberto Furuzawa), and especially to our secretaries Márcia Visone, Sônia Umeda and Angela Faria from Unifesp. This work was supported by Universal CNPq grant $n^{\circ} 477707 / 2007-6$ and co-joint Temático Fapesp grant $\mathrm{n}^{\mathrm{O}} 06 / 60402-1$.

Disclosure: we declare no affiliations that would constitute a financial conflict of interest relating to the subject matter of this study.

\section{REFERENCES}

1. Silva JE. Thermogenic mechanisms and their hormonal regulation. Physiol Rev. 2006;86(2):435-64.

2. Zhang J, Lazar MA. The mechanism of action of thyroid hormones. Annu Rev Physiol. 2000;62:439-66.

3. Brand MD, Brindle KM, Buckingham JA, Harper JA, Rolfe DF, Stuart $J A$. The significance and mechanism of mitochondrial proton conductance. Int J Obes Relat Metab Disord. 1999;23 Suppl 6:S4-11.

4. Jekabsons MB, Gregoire FM, Schonfeld-Warden NA, Warden $\mathrm{CH}$, Horwitz BA. T(3) stimulates resting metabolism and UCP-2 and UCP-3 mRNA but not nonphosphorylating mitochondrial respiration in mice. Am J Physiol. 1999;277(2 Pt 1):E380-9.

5. Silvestri E, Moreno M, Lombardi A, Ragni M, de Lange P, Alexson $\mathrm{SE}$, et al. Thyroid-hormone effects on putative biochemical pathways involved in UCP3 activation in rat skeletal muscle mitochondria. FEBS Lett. 2005;579(7):1639-45.

6. Flandin P, Lehr L, Asensio C, Giacobino JP, Rohner-Jeanrenaud F, Muzzin $P$, et al. Uncoupling protein-3 as a molecular determinant of the action of $3,5,3^{\prime}$-triiodothyronine on energy metabolism. Endocrine. 2009;36(2):246-54.

7. Shulman Gl, Ladenson PW, Wolfe MH, Ridgway EC, Wolfe RR. Substrate cycling between gluconeogenesis and glycolysis in 
euthyroid, hypothyroid, and hyperthyroid man. J Clin Invest. 1985;76(2):757-64.

8. Lombardi A, de Lange P, Silvestri E, Busiello RA, Lanni A, Goglia $F$, et al. 3,5-Diiodo-L-thyronine rapidly enhances mitochondrial fatty acid oxidation rate and thermogenesis in rat skeletal muscle: AMP-activated protein kinase involvement. Am J Physiol Endocrinol Metab. 2009;296(3):E497-502.

9. Bianco AC, Maia AL, da Silva WS, Christoffolete MA. Adaptive activation of thyroid hormone and energy expenditure. Biosci Rep. 2005;25(3-4):191-208.

10. de Meis L, Arruda AP, da Costa RM, Benchimol M. Identification of a Ca2+-ATPase in brown adipose tissue mitochondria: regulation of thermogenesis by ATP and $\mathrm{Ca} 2+$. J Biol Chem. 2006;281(24):16384-90.

11. Hannenhalli $S$, Levy S. Promoter prediction in the human genome. Bioinformatics (Oxford, England). 2001;17 Suppl 1:S90-6.

12. Levy $S$, Hannenhalli $S$, Workman C. Enrichment of regulatory signals in conserved non-coding genomic sequence. Bioinformatics (Oxford, England). 2001;17(10):871-7.

13. Venter JC, Adams MD, Myers EW, Li PW, Mural RJ, Sutton GG, et al. The sequence of the human genome. Science (New York). 2001;291(5507):1304-51.

14. Messeguer X, Escudero R, Farre D, Nunez O, Martinez J, Alba MM. PROMO: detection of known transcription regulatory elements using species-tailored searches. Bioinformatics (Oxford, England). 2002;18(2):333-4.

15. Schug J. Using TESS to predict transcription factor binding sites in DNA sequence. In: Baxevanis AD, ed. Current Protocols in Bioinformatics: J. Wiley and Sons; 2003.

16. Cartharius K, Frech K, Grote K, Klocke B, Haltmeier M, Klingenhoff $A$, et al. Matlnspector and beyond: promoter analysis based on transcription factor binding sites. Bioinformatics. 2005;21(13):2933-42.

17. Mayr B, Montminy M. Transcriptional regulation by the phosphorylation-dependent factor CREB. Nat Rev Mol Cell Biol. 2001;2(8):599-609.

18. Rousset S, del Mar Gonzalez-Barroso M, Gelly C, Pecqueur C, Bouillaud $F$, Ricquier $D$, et al. A new polymorphic site located in the human UCP1 gene controls the in vitro binding of CREB-like factor. Int J Obes Relat Metab Disord. 2002;26(5):735-8.

19. Brewer $\mathrm{C}$, Yeager N, Di Cristofano A. Thyroid-stimulating hormone initiated proliferative signals converge in vivo on the mTOR kinase without activating AKT. Cancer Res. 2007;67(17):8002-6.

20. De Luca A, Severino A, De Paolis P, Cottone G, De Luca L, De Falco $\mathrm{M}$, et al. p300/cAMP-response-element-binding-protein ('CREB')binding protein (CBP) modulates co-operation between myocyte enhancer factor 2A (MEF2A) and thyroid hormone receptor-retinoid X receptor. Biochem J. 2003;369(Pt 3):477-84.

21. Feige JN, Auwerx J. Transcriptional coregulators in the control of energy homeostasis. Trends Cell Biol. 2007;17(6):292-301.

22. Méndez-Pertuz M, Sánchez-Pacheco A, Aranda A. The thyroid hormone receptor antagonizes CREB-mediated transcription. EMBO J. 2003;22(12):3102-12.

23. Nicholls DG. The physiological regulation of uncoupling proteins. Biochim Biophys Acta. 2006;1757(5-6):459-66.

24. Farré $D$, Roset $R$, Huerta M, Adsuara JE, Rosello L, Alba MM, et al. Identification of patterns in biological sequences at the ALGGEN server: PROMO and MALGEN. Nucleic Acids Res. 2003;31(13):3651-3

25. Grabe N. AliBaba2: context specific identification of transcription factor binding sites. In Silico Biol. 2002;2(1):S1-15.

26. Matys V, Fricke E, Geffers R, Gossling E, Haubrock M, Hehl R, et al. TRANSFAC: transcriptional regulation, from patterns to profiles. Nucleic Acids Res. 2003;31(1):374-8.
27. Lin HY, Kao YH, Chen ST, Meng M. Effects of inherited mutations on catalytic activity and structural stability of human glucose-6phosphate isomerase expressed in Escherichia coli. Biochim Biophys Acta. 2009;1794(2):315-23.

28. Matsumoto N, Ishihara T, Oda E, Miwa S, Nakashima K. Fine structure of the spleen and liver in glucosephosphate isomerase (GPI) deficiency hereditary nonspherocytic hemolytic anemia. Selective reticulocyte destruction as a mechanism of hemolysis. Nippon Ketsueki Gakkai Zasshi. 1973;36(1):46-54.

29. Repiso A, Oliva B, Vives-Corrons JL, Beutler E, Carreras J, Climent F Red cell glucose phosphate isomerase (GPI): a molecular study of three novel mutations associated with hereditary nonspherocytic hemolytic anemia. Hum Mutat. 2006;27(11):1159.

30. Yoon DY, Buchler P, Saarikoski ST, Hines OJ, Reber HA, Hankinson $\mathrm{O}$. Identification of genes differentially induced by hypoxia in pancreatic cancer cells. Biochem Biophys Res Commun. 2001;288(4):882-6.

31. FunasakaT, Hogan V, Raz A. Phosphoglucose isomerase/autocrine motility factor mediates epithelial and mesenchymal phenotype conversions in breast cancer. Cancer Res. 2009;69(13):5349-56.

32. Lieberman MA, Marks A. Marks' basic medical biochemistry: a clinical approach. 3rd ed: Lippincott Williams \& Wilkins; 2009.

33. Ghayee HK, Havekes B, Corssmit EP, Eisenhofer G, Hammes SR, Ahmad Z, et al. Mediastinal paragangliomas: association with mutations in the succinate dehydrogenase genes and aggressive behavior. Endocr Relat Cancer. 2009;16(1):291-9.

34. Sparks LM, Xie H, Koza RA, Mynatt R, Hulver MW, Bray GA, et al. A high-fat diet coordinately downregulates genes required for mitochondrial oxidative phosphorylation in skeletal muscle. Diabetes. 2005;54(7):1926-33.

35. Fukuda R, Zhang H, Kim JW, Shimoda L, Dang CV, Semenza GL. HIF-1 regulates cytochrome oxidase subunits to optimize efficiency of respiration in hypoxic cells. Cell. 2007;129(1):111-22.

36. Alan L, Smolkova K, Kronusova E, Santorova J, Jezek P. Absolute levels of transcripts for mitochondrial uncoupling proteins UCP2, UCP3, UCP4, and UCP5 show different patterns in rat and mice tissues. J Bioenerg Biomembr. 2009;41(1):71-8.

37. Cunningham $O$, McElligott AM, Carroll AM, Breen $E$, Reguenga $C$, Oliveira ME, et al. Selective detection of UCP 3 expression in skeletal muscle: effect of thyroid status and temperature acclimation. Biochim Biophys Acta. 2003;1604(3):170-9.

38. Edwards $Y H$, Putt W, Fox M, Ives JH. A novel human phosphoglucomutase (PGM5) maps to the centromeric region of chromosome 9. Genomics. 1995;30(2):350-3

39. Gururaj A, Barnes CJ, Vadlamudi RK, Kumar R. Regulation of phosphoglucomutase 1 phosphorylation and activity by a signaling kinase. Oncogene. 2004;23(49):8118-27.

40. Hoffmann B, LaPaglia SK, Kubler E, Andermann M, Eckert SE, Braus GH. Developmental and metabolic regulation of the phosphoglucomutase-encoding gene, pgmB, of Aspergillus nidulans. Mol Gen Genet. 2000;262(6):1001-11.

41. Xin C, Yan-Fu W, Ping H, Jing G, Jing-Jing W, Chun-Li M, et al. Study of the insulin signaling pathways in the regulation of ACAT1 expression in cultured macrophages. Cell Biol Int. 2009;33(5):602-6.

42. Peng HM, Coon MJ. Regulation of rabbit cytochrome P450 2E1 expression in HepG2 cells by insulin and thyroid hormone. Mol Pharmacol. 1998;54(4):740-7.

43. Rosario PW. Bone and heart abnormalities of subclinical hyperthyroidism in women below the age of 65 years. Arq Bras Endocrinol Metabol. 2008;52(9):1448-51.

44. Wajner SM, Wagner MS, Maia AL. Clinical implications of altered thyroid status in male testicular function. Arq Bras Endocrinol Metabol. 2009:53(8):976-82.

45. Ryan DP, Dias da Silva MR, Soong TW, Fontaine B, Donaldson MR, Kung AW, et al. Mutations in potassium channel Kir2.6 cause susceptibility to thyrotoxic hypokalemic periodic paralysis. Cell. 2010;140(1):88-98 